Originalien

Ophthalmologe $2020 \cdot 117: 775-785$ https://doi.org/10.1007/s00347-020-01039-z Online publiziert: 5 . Februar 2020

(c) Der/die Autor(en) 2020

Christina Jacobsen 1 (D) $\cdot$ I. Volkmann' $\cdot$ F. Wedegärtner ${ }^{2} \cdot$ J. Harris $^{3} \cdot$ B. Bertram ${ }^{3} \cdot$ P. Gass ${ }^{4}$. B. Bambas ${ }^{3}$. C. Framme

'Universitätsklinik für Augenheilkunde, Medizinische Hochschule Hannover (MHH), Hannover, Deutschland

${ }^{2}$ Universitätsklinik für Psychiatrie, Sozialpsychiatrie und Psychotherapie, MHH, Hannover, Deutschland

${ }^{3}$ Berufsverband der Augenärzte Deutschlands e. V., Düsseldorf, Deutschland

${ }^{4}$ Deutsche Ophthalmologische Gesellschaft e.V., München, Deutschland

\title{
Erfahrungen von Aggression und Gewalt gegen Augenärztinnen und Augenärzte
}

liche Gewalt wie Schlagen, Anspucken oder Treten bejahten 12,7\% [4].

Der Ärztemonitor 2018 befragte im Auftrag der Kassenärztlichen Bundesvereinigung (KBV) ambulant tätige Ärzte erstmalig zu Gewalterfahrungen in ihrer beruflichen Tätigkeit. Dabei wurden auch 240 ambulant tätige Augenärzte erfasst. Von diesen schätzten nur $25 \%$, dass Ärzte/Psychotherapeuten nie angegriffen oder physisch bedroht wurden [5]. Augenärzte haben an der Spaltlampe engen körperlichen Kontakt zum Patienten und sind darüber hinaus oft mit ihren Patienten allein. Zudem wird häufig von ,längeren" Wartezeiten berichtet. Dies könnte sich negativ auf das Aggressionsverhalten von Patienten und Angehörigen auswirken [6].

Aggressionen/Gewalt im Beruf können zu emotionalem Stress führen, der u. a. Schlafstörungen, erhöhten Tabakkonsum und soziale Isolation zur Folge haben kann und als Risikofaktor für mentale Erkrankungen gilt [7]. Eine Studie aus Frankreich erfasste die Gewalterfahrungen der Mitarbeiter für eine augenärztliche Notfallabteilung. Als Gründe, die zu Aggressionen/Gewalt führten, wurden lange Wartezeiten, fehlende $\mathrm{Pa}$ tienteninformationen sowie Unterbesetzung genannt. Die Befragten waren sogar der Ansicht, dass das permanente Unterbrechen der Arbeit durch Patientenbeschwerden belastender sei als tatsächliche Gewalt [8].
Aufgrund der Aktualität des Themas und der bescheidenen Datenlage führten wir in Zusammenarbeit mit dem Berufsverband für Augenärzte (BVA) und der Deutschen Ophthalmologischen Gesellschaft (DOG) eine Befragung unter den deutschen Augenärzten durch. Ziel war es, eine Datengrundlage zu schaffen, möglichen Handlungsbedarf zu identifizieren und ggf. geeignete Präventionsmaßnahmen zu entwickeln.

\section{Methodik}

Auf Grundlage der Aggressions-Wahrnehmungsskala (POPAS Fragebogen; [9]) sowie der Umfrage zu Aggressionen und Gewalt unter Allgemeinmedizinern [3] wurde ein Fragebogen für Augenärzte entwickelt (•Tab. 4 im Anhang). Im Herbst 2018 erhielten alle Mitglieder von DOG und BVA per E-Mail eine personalisierte Einladung (Token) mit einem Link, um den Fragebogen online auszufüllen. Durch Datenabgleich wurde vermieden, dass Personen, die beiden Verbänden als Mitglieder angehören, doppelt angeschrieben wurden. Vor jeder Befragung wurde die Einwilligung für die Teilnahme an dem Projekt abgefragt sowie über die datenschutzrechtlichen Maßnahmen aufgeklärt. Den Teilnehmern war es möglich, die personalisierte Befragung zu unterbrechen und zu einem späteren Zeitpunkt fortzuführen. Die Daten wurden nach Abschluss der Befragung vollständig anonymisiert und 
das Token als benutzt gewertet. Insgesamt wurden 3 Reminder mit der Bitte um Beteiligung an der Umfrage an die jeweils noch offenen Token versendet. Die Befragung selbst wurde über die Webplattform Soscisurvey (SoSci Survey GmbH, München, www. soscisurvey.de, Server: sosci01.mh-hannover.local, Version 3.2.00) über einen eigenen Hochschulserver realisiert.

Die statistische Auswertung und die Generierung von Tabellen, Grafiken und Datenlisten wurden mit dem statistischen Programm SPSS Version 25.0 (Chicago, IL, USA) sowie Excel (Version 2010, Microsoft, Redmont, USA) durchgeführt. Es wurden Subgruppen für verschiedene Erfahrungen gebildet. Zur allgemeinen deskriptiven statistischen Auswertung von Häufigkeiten wurden Mittelwert, Standardabweichung der Stichprobe, Median, Minimum, Maximum und Häufigkeitsangaben in Prozent verwendet. Sechs Extremwerte mit Häufigkeitsangaben größer 500 für erlebte Gewalt innerhalb der letzten zwölf Monate wurden herausgenommen, da diese unrealistisch hoch waren. Für nominalskalierte Angaben wurde der ChiQuadrat-Test nach Pearson verwendet. Das Signifikanzniveau betrug $5 \%$.

\section{Ergebnisse}

Von den 9411 angeschriebenen Augenärzten (einschließlich Ärzte in der Weiterbildung) nahmen insgesamt 1508 (Alter: $49 \pm 12$ Jahre) Ärzte an der Befragung teil $(16 \%)$. Von diesen waren 806 (53,7\%) Teilnehmer weiblich. 751 (49,8\%) Augenärzte gehörten sowohl der DOG als auch dem BVA an. 462 $(30,6 \%)$ waren nur BVA-Mitglieder und $268(17,8 \%)$ waren nur DOG-Mitglieder. 1139 (75,5\%) Teilnehmer arbeiteten in Praxen. 1020 (68,3\%) Ärzte gaben als aktuelle Funktion Facharzt/-ärztin an. Entsprechend der Einwohnerzahl waren die meisten Augenärzte in NordrheinWestfalen tätig. 1264 (83,8\%) Augenärzte gaben an, in ihrer augenärztlichen Tätigkeit bereits Aggressionen/Gewalt erlebt zu haben ( $\bullet$ Tab. 1).

Augenärztinnen berichteten im Vergleich zu den männlichen Kollegen signifikant häufiger, Aggressionen/Gewalt erfahren zu haben $(p=0,001)$. Augenärztinnen erlebten insbesondere sexuelle Einschüchterungen/Übergriffe signifikant häufiger als die männlichen Kollegen $(p<0,001)$. Für körperliche Gewalt existierte kein signifikanter Unterschied zwischen den Geschlechtern $(p=0,381)$.

Augenärzte, die aktuell nicht tätig waren und sich z. B. in Elternzeit befanden oder bereits in der Rente waren, erlebten laut der Erhebung deutlich weniger Aggressionen/Gewalt als aktive Augenärzte. Assistenzärzte erlebten signifikant häufiger Aggressionen/Gewalt als erfahrenere Ärzte $(p<0,001)$.

Augenärzte, die in Städten mit mehr als 50.000 Einwohnern arbeiteten, erlebten signifikant häufiger körperliche Gewalt $(p=0,001)$ sowie sexuelle Einschüchterung/Übergriffe $(p=0,002)$ als Ärzte, die in Städten mit weniger als 50.000 Einwohnern arbeiteten. Für verbale Gewalt $(p=0,726)$ und aggressives Verhalten $(p=0,464)$ existierte kein signifikanter Unterschied.

\section{Muttersprache und Migrations- hintergrund}

Insgesamt gaben 155 (10,3\%) Ärzte an, dass Deutsch nicht ihre Muttersprache sei. Dabei zeigte sich eine regelrechte Sprachenvielfalt von insgesamt 29 Sprachen. 16 (10,3\%) Ärzte gaben Russisch und 14 (9\%) Ärzte Arabisch als ihre Muttersprache an. Interessanterweise hatten „nur“ 117 (75,5\%) Ärzte, die nicht Deutsch als Muttersprache angaben, Aggressionen/Gewalt erlebt und hatten somit signifikant $(p=0,003)$ weniger Aggressionen/Gewalt als deutsche Muttersprachler $(84,3 \%)$ erfahren. Bei verbaler Gewalt $(p=0,031)$ sowie aggressivem Verhalten $(p=0,002)$ zeigte sich ein signifikanter Unterschied. Ärzte mit deutscher Muttersprache machten entsprechend mehr Erfahrungen oder gaben dies zumindest an.

Einen Migrationshintergrund hatten 232 (15,4\%) Ärzte. Es existierte kein signifikanter Unterschied bezüglich Aggressionen/Gewalt insgesamt aufgrund eines Migrationshintergrunds $(p=0,463)$. Aggressives Verhalten erlebten Ärzte mit einem Migrationshintergrund jedoch signifikant weniger $(p=0,046)$ als Ärzte ohne Migrationshintergrund. 91 (6,1\%) Ärzte hatten den Eindruck, dass sie aufgrund ihrer Sprache/Herkunft Ziel von Patientenaggressionen wurden. Davon hatten 43 (18,5\%) Ärzte Migrationshintergrund, und 34 (22\%) Ärzte waren keine deutschen Muttersprachler. In der Gruppe ohne Migrationshintergrund bzw. Deutsch als Muttersprache gaben jeweils unter $4 \%$ die Sprache/Herkunft als Ursache von Patientenaggressionen an.

- Tab. 2 stellt zu der jeweiligen Gewalterfahrung die entsprechenden Mittelwerte für die letzten 12 Monate dar. Am häufigsten wurden verbale Übergriffe ohne Drohung erlebt. Der Median mit 5 Vorfällen im Jahr war bei spaltend aggressivem Verhalten am höchsten. Überraschend hoch ist die Angabe von 27 $(1,8 \%)$ Augenärzten, die einen vollendeten Suizid während der Konsultation erlebt haben.

\section{Ärzte in der Klinik im Vergleich zur Praxis}

$235(81,3 \%)$ von 289 Ärzten, die in Kliniken tätig sind, erlebten Gewalt v. a. in Kliniken, und 896 (79,6\%) von 1125 Ärzten aus Praxen erfuhren Gewalt v. a. in Praxen. In der Gruppe ohne aktuelle Beschäftigung hatten nur 38 (50\%) von 76 Ärzten überhaupt Gewalterfahrungen gemacht, von denen 29 (38,2\%) Ärzte diese eher in Praxen gemacht haben.

\section{Arbeit im Notdienst}

1118 (92,5\%) Ärzte gaben an, den größten Teil der oben genannten Erfahrungen in der regulären Arbeitszeit erlebt zu haben. 1075 (71,3\%) Ärzte nehmen am Notdienst am Wochenende oder außerhalb der regulären Arbeitszeit teil. In der am Notdienst teilnehmenden Ärztegruppe gaben 824 (77,2 \%) Ärzte an, dass sich die Vorfälle v. a. in der regulären Arbeitszeit ereigneten, im Vergleich zu 294 $(69,3 \%)$ Ärzte in der Gruppe, die nicht am Notdienst teilnehmen. Augenärzte, die am Notdienst teilnehmen, hatten jedoch signifikant häufiger Aggressionen/ Gewalt erfahren $(p<0,001)$.

Im Notdienst sind 572 (53,3\%) Ärzte allein, während $345(32,1 \%)$ von einer 
Ophthalmologe 2020 $\cdot 117: 775-785$ https://doi.org/10.1007/s00347-020-01039-z

(c) Der/die Autor(en) 2020

\section{Jacobsen · I. Volkmann · F. Wedegärtner · J. Harris · B. Bertram · P. Gass · B. Bambas · C. Framme}

\section{Erfahrungen von Aggression und Gewalt gegen Augenärztinnen und Augenärzte}

\section{Zusammenfassung}

Hintergrund. Die Medien berichten

über die erhöhte Gewaltbereitschaft von

Patienten sowie Angehörigen gegenüber

medizinischem Personal. Bislang wurden wenige Untersuchungen zu diesem Thema durchgeführt, entsprechend schwach ist die Datenlage. Gerade Augenärzte haben an der Spaltlampe engen Kontakt zum Patienten und sind oft mit Patienten allein.

Methodik. Auf Grundlage der AggressionsWahrnehmungsskala (POPAS Fragebogen, Oud 2000) sowie der Umfrage zu Aggressionen und Gewalt unter Allgemeinmedizinern (Vorderwülbecke et al. 2015) wurde ein Fragebogen entwickelt. Im Herbst 2018 erhielten alle Mitglieder der Deutschen Ophthalmologischen Gesellschaft (DOG) und des Berufsverbandes der Augenärzte (BVA) per Mail eine Einladung, um den Fragebogen online auszufüllen.

Ergebnisse. Von 9411 angeschriebenen Augenärzten nahmen insgesamt 1508 (Alter: $49 \pm 12$ Jahre) an der Befragung teil (16\%). 806 (53,7\%) Befragte waren weiblich. 1139 (75,5\%) Teilnehmer arbeiteten in Praxen. Insgesamt 1264 (83,3\%) der Augenärzte hatten Aggressionen/Gewalt in ihrer Tätigkeit erfahren. 986 (65\%) Befragte hatten verbale Übergriffe ohne Drohung erlebt. Von bedrohlich körperlichen Gewalterfahrungen berichteten 363 (24,1\%) Ärzte. 30 (2\%) Teilnehmer erhielten aufgrund schwerer körperlicher Gewalt eine ärztliche Behandlung. Sexuelle Einschüchterung/Belästigung bejahten 322 (21,4\%) der Befragten, von diesen waren 243 (75,5\%) weiblich. 533 (47,9\%) Ärzte empfanden, dass aggressive/gewalttätige Verhaltensweisen in den letzten 5 Jahren zugenommen haben. Schlussfolgerung. Die hohe Teilnehmeranzahl weist auf die hohe Relevanz des Themas hin. Die Umfrage hat erstaunliche Ergebnisse geliefert, die weitere Diskussionen und Handlungen zur Folge haben sollten, um die Sicherheit der Mitarbeiter zu verbessern.

\section{Schlüsselwörter}

Gewalt · Aggression · Mitarbeiterzufriedenheit · Arbeitssicherheit · Bedrohung

\section{Experiences of aggression and violence against ophthalmologists}

\begin{abstract}
Background. The media have reported an increased willingness of patients and relatives to use violence against medical personnel. So far a few studies have been carried out on this topic and the data situation is correspondingly weak. Ophthalmologists in particular have close contact with patients at the slit lamp and are often alone with patients.

Methods. A questionnaire was developed based on the perception of prevalence of aggression scale (POPAS) questionnaire and the survey on aggression and violence among general practitioners. In autumn 2018, all members of the German Ophthalmological Society (DOG) and the Professional Associ-
\end{abstract}

ation of Ophthalmologists (BVA) received an invitation by e-mail to complete the questionnaire online.

Results. Of the 9411 ophthalmologists contacted a total of 1508 (age $49 \pm 12$ years) took part in the survey (16\%). Of the respondents 806 (53.7\%) were female and 1139 (75.5\%) participants worked in practices. A total of 1264 (83.3\%) ophthalmologists had experienced aggression/violence in their work, $986(65 \%)$ respondents had already experienced verbal assaults without threats, $363(24.1 \%)$ doctors reported experiences with threats of physical violence and 30 (2\%) participants had received medical treatment for severe physical violence. Sexual intimidation/harassment was affirmed by 322 (21.4\%) of respondents, of whom 243 (75.5\%) were female and $533(47.9 \%)$ doctors felt that aggressive/violent behavior had increased in the last 5 years.

Conclusion. The high number of participants indicates the high relevance of the topic. The survey produced astonishing results, which should lead to further discussion and action to improve the safety of employees.

Keywords

Violence · Aggression - Employee satisfaction . Work safety - Threats medizinischen Fachkraft unterstützt werden. Nur 58 (5,4\%) Ärzte werden von einem ärztlichen Kollegen unterstützt. 38 (3,5\%) Ärzte sind zeitweise allein. Im Notdienst in einer Klinik bzw. Notfallpraxis sind 27 (2,5\%) Ärzte tätig. 11 (1\%) Ärztinnen werden vom Partner/ Ehemann begleitet. Im Notdienst warteten die Patienten bei 872 (81,5\%) Ärzten unter $60 \mathrm{~min}$, davon arbeiteten 731 $(83,8 \%)$ Ärzte in Praxen. Bei Wartezeiten über 60 min waren nur $90(45,5 \%)$ von 198 Augenärzten in Praxen tätig. Mit zunehmender durchschnittlicher Wartezeit stieg der Anteil von Ärzten mit Erfah- rungen von Aggressionen/Gewalt an. Er betrug bei über $120 \mathrm{~min}$ über $95 \%$.

\section{Veränderung von Gewalt- erfahrungen}

Insgesamt fanden 533 (35,5\%) Ärzte, dass aggressive Verhaltensweisen in den letzten 5 Jahren zugenommen haben. Dabei empfanden v. a. Ärzte, die Aggressionen/Gewalt erfahren haben, dass aggressive Verhaltensweisen zugenommen haben (• Abb. 1).

\section{Medizinisches Assistenzpersonal}

Der Fragebogen wurde nur vom ärztlichen Personal beantwortet. 1047 (69,6\%) Ärzte gaben an, dass das Assistenzpersonal Aggressionen/Gewalt durch Patienten erfahren habe. Dabei beurteilten 346 (29,0\%) Ärzte verbale Übergriffe ohne Drohung als die schlimmsten Vorfälle. Körperliche Gewalt wurde von 48 (4\%) Ärzten als schlimmste Gewalterfahrung bejaht. 950 (76,6 \%) Ärzte hatten den Eindruck, dass das Assistenzpersonal mehr Gewalt als das ärztliche Personal erfahre. 
Tab. 1 Charakteristika der teilnehmenden Augenärzte

\begin{tabular}{|l|l|l|l|l|}
\hline $\begin{array}{l}\text { Gesamt Teil- } \\
\text { nehmer }\end{array}$ & $\begin{array}{l}\text { Aggressionen/ } \\
\text { Gewalt erlebt } \\
\text { gesamt }\end{array}$ & $\begin{array}{l}\text { Verbale Ge- } \\
\text { walt erlebt }\end{array}$ & $\begin{array}{l}\text { Aggressives } \\
\text { Verhalten } \\
\text { erlebt }\end{array}$ & $\begin{array}{l}\text { Körperliche } \\
\text { Gewalt erlebt }\end{array}$ \\
\hline
\end{tabular}

Sexuelle Eingesamt

erlebt

schüchterung/

Übergriffe

erlebt

$\begin{array}{llllllllllll}\% & n & \% & n & \% & n & \% & n & \% & n & \% & n\end{array}$

Welches Geschlecht haben Sie?

Männlich

Weiblich

$53,7 \quad 806$

Gesamt

In welcher Einrichtung arbeiten Sie aktuell?

Einzelpraxis

Gemeinschaftspraxis

MVZ

Klinik

Universitätsklinik

Ohne Arbeit (Rentner/Pensionär/

Elternzeit etc.)

Gesam

Welche Funktion haben Sie aktuell?

Assistenzarzt/-ärztin

Im 1. oder 2. Weiterbildungsjahr

Assistenzarzt/-ärztin

Ab dem 3. Weiterbildungsjahr

Facharzt/-ärztin

Oberarzt/-ärztin

Leitender Oberarzt/-ärztin

Chefarzt/-ärztin

Ohne Arbeit (Rentner/Pensionär/

Elternzeit etc.)

Gesamt
In welchem Bundesland arbeiten Sie?
Baden-Württemberg
Bayern
Berlin
Brandenburg
Bremen
Hamburg
Hessen
Mecklenburg-Vorpommern
Niedersachsen
Nordrhein-Westfalen
Rheinland-Pfalz
Saarland
Sachsen
Sachsen-Anhalt
Schleswig-Holstein
Thüringen
Gesamt

\begin{tabular}{|c|c|c|c|c|c|c|c|c|c|c|c|}
\hline 32,3 & 487 & 88,3 & 430 & 74,7 & 364 & 79,5 & 387 & 5,4 & 26 & 21,1 & 103 \\
\hline 33,2 & 501 & 84,2 & 422 & 62,9 & 313 & 77,0 & 386 & 6,0 & 30 & 18,6 & 93 \\
\hline 10 & 151 & 82,8 & 125 & 64,2 & 97 & 74,8 & 113 & 7,9 & 12 & 25,8 & 39 \\
\hline 9,6 & 145 & 86,2 & 125 & 71,0 & 103 & 75,2 & 109 & 10,3 & 15 & 25,5 & 37 \\
\hline 9,7 & 146 & 83,2 & 122 & 65,8 & 96 & 74,0 & 108 & 11,6 & 17 & 28,1 & 41 \\
\hline 5,1 & 77 & 21,9 & 40 & 33,8 & 26 & 41,6 & 32 & 0 & 0 & 14,3 & 11 \\
\hline
\end{tabular}

$\begin{array}{llllllllllll}100 & 1507 & 83,9 & 1264 & 66,4 & 999 & 75,3 & 1135 & 6,7 & 100 & 21,5 & 324\end{array}$

$\begin{array}{llllllllllll}2,2 & 33 & 97 & 32 & 75,8 & 25 & 87,9 & 29 & 6,1 & 2 & 33,3 & 11\end{array}$

$\begin{array}{llllllllllll}8,1 & 121 & 90,1 & 109 & 76,9 & 93 & 77,7 & 94 & 12,4 & 15 & 37,2 & 45\end{array}$

$\begin{array}{llllllllllll}68,3 & 1020 & 85,3 & 870 & 68,1 & 693 & 77,5 & 790 & 6,0 & 61 & 20,5 & 209\end{array}$

$\begin{array}{llllllllllll}6,4 & 95 & 84,2 & 80 & 62,1 & 59 & 77,9 & 74 & 9,5 & 9 & 15,8 & 15\end{array}$

$\begin{array}{llllllllllll}2,1 & 32 & 84,4 & 27 & 56,3 & 18 & 68,8 & 22 & 6,3 & 2 & 40,6 & 13\end{array}$

$\begin{array}{llllllllllll}7,2 & 107 & 85 & 91 & 67,3 & 72 & 78,5 & 84 & 8,4 & 9 & 16,8 & 18\end{array}$

$\begin{array}{llllllllllll}5,8 & 86 & 50 & 43 & 32,6 & 28 & 39,5 & 34 & 1,2 & 1 & 14,0 & 12\end{array}$

$\begin{array}{llllllllllll}100 & 1494 & 83,8 & 1252 & 66,3 & 988 & 75,4 & 1127 & 6,7 & 99 & 21,6 & 323\end{array}$

$\begin{array}{llllllllllll}13,2 & 193 & 83,9 & 162 & 66,8 & 129 & 77,2 & 149 & 5,7 & 11 & 20,2 & 39\end{array}$

$\begin{array}{llllllllllll}14,1 & 206 & 88,3 & 182 & 66 & 136 & 79,6 & 164 & 6,4 & 13 & 27,2 & 56\end{array}$

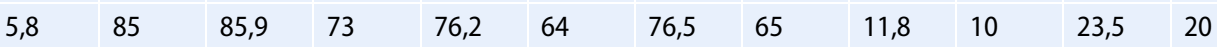

$\begin{array}{llllllllllll}2,4 & 35 & 88,6 & 31 & 74,3 & 26 & 71,4 & 25 & 0 & 0 & 17,1 & 6\end{array}$

$\begin{array}{llllllllllll}1 & 15 & 86,7 & 13 & 73,3 & 11 & 80,0 & 12 & 0 & 0 & 26,7 & 4\end{array}$

$\begin{array}{llllllllllll}2,9 & 42 & 83,3 & 35 & 61,9 & 26 & 73,8 & 31 & 11,9 & 5 & 33,3 & 14\end{array}$

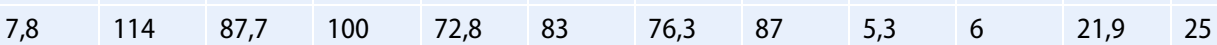

$\begin{array}{llllllllllll}1,5 & 22 & 68,2 & 15 & 59,1 & 13 & 54,5 & 12 & 0 & 0 & 13,6 & 3\end{array}$

$\begin{array}{lllllllllllll}8,9 & 130 & 86,2 & 112 & 68,2 & 88 & 80,0 & 104 & 8,5 & 11 & 22,3 & 29\end{array}$

$\begin{array}{llllllllllll}23,7 & 346 & 81,2 & 281 & 65,6 & 227 & 73,7 & 255 & 7,5 & 26 & 17,6 & 61\end{array}$

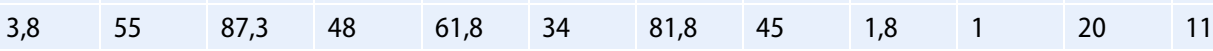

$\begin{array}{lllllllllllll}1,2 & 18 & 77,8 & 14 & 50 & 9 & 66,7 & 12 & 5,6 & 1 & 22,2 & 4\end{array}$

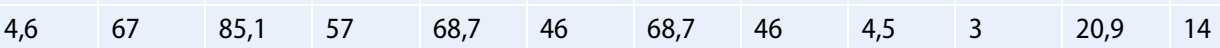

$\begin{array}{lllllllllllll}2,9 & 43 & 83,7 & 36 & 76,7 & 33 & 79,1 & 34 & 4,7 & 2 & 16,3 & 7\end{array}$

$\begin{array}{lllllllllllll}4,2 & 61 & 85,2 & 52 & 76,6 & 33 & 77,0 & 47 & 3,3 & 2 & 19,7 & 12\end{array}$

$\begin{array}{llllllllllll}2,1 & 30 & 76,6 & 23 & 70 & 21 & 70 & 21 & 20 & 6 & 23,3 & 7\end{array}$

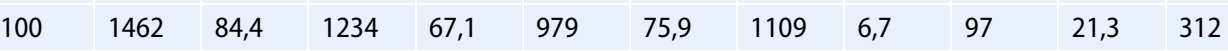




\begin{tabular}{|c|c|c|c|c|c|c|c|c|c|c|c|c|}
\hline & \multicolumn{2}{|c|}{$\begin{array}{l}\text { Gesamt Teil- } \\
\text { nehmer }\end{array}$} & \multicolumn{2}{|c|}{$\begin{array}{l}\text { Aggressionen/ } \\
\text { Gewalt erlebt } \\
\text { gesamt }\end{array}$} & \multicolumn{2}{|c|}{$\begin{array}{l}\text { Verbale Ge- } \\
\text { walt erlebt }\end{array}$} & \multicolumn{2}{|c|}{$\begin{array}{l}\text { Aggressives } \\
\text { Verhalten } \\
\text { erlebt }\end{array}$} & \multicolumn{2}{|c|}{$\begin{array}{l}\text { Körperliche } \\
\text { Gewalt erlebt }\end{array}$} & \multicolumn{2}{|c|}{$\begin{array}{l}\text { Sexuelle Ein- } \\
\text { schüchterung/ } \\
\text { Übergriffe } \\
\text { erlebt }\end{array}$} \\
\hline & $\%$ & $n$ & $\%$ & $n$ & $\%$ & $n$ & $\%$ & $n$ & $\%$ & $n$ & $\%$ & $n$ \\
\hline \multicolumn{13}{|c|}{ Arbeiten Sie in einer Stadt mit mehr als 50.000 Einwohnern? } \\
\hline Ja & 65,5 & 972 & 83,3 & 810 & 66,5 & 646 & 74,7 & 726 & 8,1 & 79 & 24,0 & 233 \\
\hline Nein & 34,5 & 513 & 84,8 & 435 & 65,6 & 336 & 76,4 & 392 & 3,7 & 19 & 17,0 & 87 \\
\hline Gesamt & 100 & 1485 & 83,3 & 1245 & 66,2 & 982 & 75,3 & 1118 & 6,6 & 98 & 21,5 & 320 \\
\hline \multicolumn{13}{|c|}{ Haben Sie einen Migrationshintergrund? } \\
\hline Ja & 15,4 & 232 & 82,3 & 191 & 64,2 & 149 & 70,3 & 163 & 6,9 & 16 & 20,3 & 47 \\
\hline Nein & 84,6 & 1270 & 84,3 & 1070 & 66,9 & 848 & 76,4 & 970 & 6,6 & 83 & 21,8 & 277 \\
\hline Gesamt & 100 & 1502 & 84 & 1261 & 66,5 & 997 & 75,4 & 1133 & 6,6 & 99 & 21,6 & 324 \\
\hline \multicolumn{13}{|c|}{ Ist Deutsch Ihre Muttersprache? } \\
\hline $\mathrm{Ja}$ & 89,7 & 1346 & 84,4 & 1142 & 67,4 & 905 & 76,4 & 1029 & 6,7 & 90 & 21,9 & 295 \\
\hline Nein & 10,3 & 155 & 75,5 & 117 & 58,7 & 91 & 65,2 & 101 & 6,5 & 10 & 18,1 & 28 \\
\hline Gesamt & 100 & 1501 & 83,9 & 1259 & 66,4 & 996 & 75,3 & 1130 & 6,7 & 100 & 21,5 & 323 \\
\hline
\end{tabular}

Tab. 2 Angaben zu Gewalterfahrungen jemals und innerhalb der letzten 12 Monate Gewalterfahrungen und Beispiele

\begin{tabular}{|c|c|c|c|c|}
\hline & \multirow{2}{*}{$\begin{array}{l}\text { erlebt } \\
\%(n)\end{array}$} & \\
\hline & & Mittelwert & Median & $\begin{array}{l}\text { Standard- } \\
\text { abweichung }\end{array}$ \\
\hline $\begin{array}{l}\text { Verbale Übergriffe ohne Drohung: Beleidigungen, Fluchen, Beschimpfungen, Herum- } \\
\text { brüllen, persönliche Beleidigungen, Schreien }\end{array}$ & $65,6(986)$ & 10,7 & 3 & 25,2 \\
\hline $\begin{array}{l}\text { Bedrohendes verbales Verhalten: boshaft Fluchen, verbale Drohung von Gewalt, Wutan- } \\
\text { fälle, Androhung von Gewalt außerhalb des Arbeitsplatzes }\end{array}$ & $23,7(355)$ & 5,3 & 2 & 23,3 \\
\hline $\begin{array}{l}\text { Demütigendes aggressives Verhalten: eindeutige persönliche Beleidigungen, ausfällig } \\
\text { Fluchen, Spucken, abwertende Bemerkungen und Gesten }\end{array}$ & $28,5(427)$ & 4,5 & 2 & 10,9 \\
\hline $\begin{array}{l}\text { Herausforderndes aggressives Verhalten: Provokation oder Verhalten, das zu negativer } \\
\text { Reaktion führt }\end{array}$ & $41,1(613)$ & 9,1 & 3 & 28,1 \\
\hline Passiv aggressives Verhalten: Verhalten, das irritierend, störend, blockierend wirkt & $59,1(880)$ & 12,4 & 4 & 33,2 \\
\hline $\begin{array}{l}\text { Spaltend aggressives Verhalten: manipulatives Verhalten, das zu Streit oder Disharmo- } \\
\text { nie beim Personal führt }\end{array}$ & $49,5(730)$ & 16,6 & 5 & 47,9 \\
\hline $\begin{array}{l}\text { Bedrohlich körperliches Verhalten: Schlagen von Türen, Werfen und Treten von Gegen- } \\
\text { ständen, Bedrohen mit Waffen }\end{array}$ & $24,3(363)$ & 3,3 & 2 & 5,8 \\
\hline $\begin{array}{l}\text { Zerstörerisch aggressives Verhalten: Beschädigen, Zerstören oder Zerschlagen von } \\
\text { Gegenständen }\end{array}$ & $8,3(124)$ & 1,4 & 1 & 1,9 \\
\hline $\begin{array}{l}\text { Mäßige körperliche Gewalt: Beißen, Schlagen, Treten, Würgen, Austeilen von Fausthie- } \\
\text { ben, Kratzen, an den Haaren Ziehen oder Ausreißen }\end{array}$ & $6,6(99)$ & 1,4 & 1 & 2,6 \\
\hline $\begin{array}{l}\text { Schwere körperliche Gewalt: schwere Verletzungen, die zu einer ärztlichen Behandlung } \\
\text { führten (z. B. Knochenbrüche, Bewusstlosigkeit oder Fleischwunden) }\end{array}$ & $2,0(30)$ & 0,1 & 0 & 0,3 \\
\hline $\begin{array}{l}\text { Mäßige gegen sich selbst gerichtete Gewalt: Patientlnnen, die sich selbst kratzen, bei- } \\
\text { ßen, schlagen etc., mit keinen oder nur kleineren Verletzungen (während der Konsultati- } \\
\text { on) }\end{array}$ & $6,8(101)$ & 1,7 & 1 & 1,9 \\
\hline $\begin{array}{l}\text { Schwere gegen sich selbst gerichtete Gewalt: behandlungsbedürftige Selbstverstüm- } \\
\text { melung, tiefe Schnitte, Bisse, Verbrennungen, innere Verletzungen, Brüche, Bewusstlo- } \\
\text { sigkeit (während der Konsultation) }\end{array}$ & $2,8(42)$ & 0,5 & 0 & 0,9 \\
\hline $\begin{array}{l}\text { Versuchter Suizid: Einnahme einer Medikamentenüberdosis, Aufschneiden der Puls- } \\
\text { adern, Sprung von Gebäuden (während der Konsultation) }\end{array}$ & $2,5(37)$ & 0,3 & 0 & 0,5 \\
\hline Vollendeter Suizid: (während der Konsultation) & $1,8(27)$ & 0,1 & 0 & 0,3 \\
\hline $\begin{array}{l}\text { Sexuelle Einschüchterung/Belästigung: obszöne Gesten, exhibitionistische Übergriffe, } \\
\text { zweideutige Bemerkungen, private Kontaktaufnahme, sexistische Verhaltensweisen }\end{array}$ & $21,6(322)$ & 3,9 & 2 & 10,0 \\
\hline Sexueller Übergriff: sexuelle Nötigung, Vergewaltigung & $2,0(30)$ & 0,1 & 0 & 0,3 \\
\hline
\end{tabular}


Tab. 3 Altersverteilung nach Geschlechtern und Gewalterfahrungen (Mittelwert \pm Standardabweichung in Jahren)

\begin{tabular}{llll} 
Geschlecht & $\begin{array}{l}\text { Aggression/Gewalt } \\
\text { erfahren }\end{array}$ & $\begin{array}{l}\text { Keine Aggression/ } \\
\text { Gewalt erfahren }\end{array}$ & Gesamtergebnis \\
\hline Männlich & $51,1 \pm 11,5$ & $56,6 \pm 13,6$ & $52,1 \pm 12,1$ \\
Weiblich & $45,7 \pm 10,5$ & $50,6 \pm 12,8$ & $46,3 \pm 11$ \\
Gesamt & $48,1 \pm 11,2$ & $55 \pm 13,6$ & $49 \pm 11,8$
\end{tabular}

\section{Gewalterfahrungen abhängig vom} Alter

Im Mann-Whitney-U-Test $(p<0,001)$ zeigte sich ein signifikanter Unterschied bezüglich des Alters und des Geschlechts der Umfrageteilnehmer hinsichtlich jemals erfahrener Gewalt (• Tab. 3).

\section{Gegen Gewalt getroffene Vorkehrungen oder Maßnahmen}

Die Ärzte wurden nach Vorkehrungen/Maßnahmen gegen Gewalt gefragt (• Abb. 2). Insgesamt gaben 819 (54,3\%) Ärzte an, keine Maßnahmen getroffen zu haben. 421 (28\%) Ärzte hatten Maßnahmen zum Konfliktmanagement für Ärzte/Mitarbeiter wie interne Schulungen oder Leitlinien eingeführt.

\section{Diskussion}

Mit über 1500 Teilnehmern bei 9411 angeschriebenen Augenärzten handelt es sich um eine sehr hohe Teilnahmequote. Dies unterstreicht die Relevanz des Themas in der Augenheilkunde. Neben häufiger verbaler Gewalt kam es auch zu gravierenden Vorfällen, wenn zum Beispiel Assistenzärzten ein Revolver an die Stirn gehalten wurde, weil die Schmerzen bei der Diagnose einer Erosio corneae nicht schnell genug beseitigt wurden, oder als Patienten getarnte Täter, die einen bewaffneten Raubüberfall begingen. Insgesamt ist der Anteil von Augenärzten in der Umfrage, die Aggressionen/Gewalt jemals in ihrer Tätigkeit erfahren haben, mit 83,3\% sehr hoch. Dabei gaben Augenärztinnen und junge Ärztinnen und Ärzte signifikant häufiger entsprechende Erfahrungen an. In der Umfrage unter Allgemeinmedizinern hatten sogar nur 9\% der Teilnehmer nie in ihrer Laufbahn aggressives Verhalten erlebt [3].
Aggressionen können als Zeichen von Stress oder unerfüllten Bedürfnissen gesehen werden. Ursachen für Gewalt im Gesundheitssektor können sowohl intrinsisch als auch extrinsisch bedingt sein. Intrinsische Faktoren sind unter anderem psychische Grunderkrankungen, bei denen in einer alternden Gesellschaft insbesondere die Demenz hervorzuheben ist. Auch Drogen- und Alkoholabhängigkeit sind $\mathrm{zu}$ beachten. Als extrinsische Faktoren zählen das Verhalten des Personals und die Versorgungssituation [10]. In der Augenheilkunde kommt es aktuell zu einem zunehmenden Versorgungsengpass aufgrund der Zunahme von altersbedingten Augenerkrankungen und einer zugleich geringen Zunahme der Versorgungskapazität [11]. Dies führt zu längeren Wartezeiten und kann auch zu einem kürzeren Arzt-Patienten-Kontakt führen, wodurch sich die Unzufriedenheit der Patienten steigern kann. Nicht $\mathrm{zu}$ vergessen ist der Umstand, dass unter stressigen Arbeitsbedingungen das Konfliktpotenzial auch seitens der Mitarbeiter steigt [12].

Über 60-jährige Ärzte und Psychotherapeuten $(30 \%)$ erlebten laut Ärztemonitor 2018 weniger verbale Gewalt als jüngere Ärzte (43\%). Körperliche Gewalt/physische Bedrohung in den letzten 12 Monaten widerfuhr $27 \%$ der unter 44 Jährigen. Ab 50 Jahren betrug der Anteil nur noch $15 \%$ [5]. Zu beachten ist, dass der Ärztemonitor 2018 nicht den POPAS Fragebogen als Grundlage verwendete und nur wenige Fragen zur Gewalt erfasste. Dies kann die verschiedenen Prozentzahlen begründen. Auch in unserer Umfrage zeigte sich ein signifikanter Altersunterschied zulasten der jüngeren Ärzte. Eine Studie aus Indien arbeitete als Risikofaktoren für Gewalt ein junges Alter heraus [13]. Eine mögliche Erklärung ist, dass Patienten älteren Ärzten mehr Respekt entgegenbringen.
Auch ist anzunehmen, dass erfahrenere Ärzte Situationen mit aggressivem Verhalten aufgrund ihrer längeren Berufserfahrung besser handhaben können oder sie als weniger bedrohlich oder belastend wahrnehmen.

In unserer Umfrage zeigte sich recht eindrücklich, dass Augenärztinnen mit Ausnahme von körperlicher Gewalt signifikant häufiger Aggressionen/Gewalt erlebten als ihre männlichen Kollegen (87\% zu 80,4\%). Dies präsentierte bereits die Analyse von Kumar et al. [13]. Im Ärztemonitor 2018 berichteten $42 \%$ der Frauen im Vergleich zu 36\% der Männer, verbale Gewalt innerhalb der letzten 12 Monate erfahren zu haben [5]. Mit 30,3\% ist der Anteil von Ärztinnen in dieser Studie, die sexuelle Einschüchterung/Übergriffe erlebt haben, erschreckend hoch. In einer Umfrage in den USA und Kanada hatten sogar 59\% der teilnehmenden Augenärztinnen sexuelle Belästigung erlebt [14]. Hierbei müssen jedoch Kulturunterschiede bedacht werden. Auch in den Freitextfeldern zeigte sich, dass viele Augenärztinnen sich unsicher fühlten, wenn sie während des Notdienstes alleine waren bzw. darüber berichteten, dass sie aus Präventionsgründen ihre Partner mit einbezogen. Weitere Untersuchungen zu diesem besonderen Umstand erscheinen sinnvoll und wichtig.

Im Ärztemonitor 2018 empfanden $38 \%$ der Augenärzte, dass die Gewalt zugenommen habe [5]. In unserer Umfrage sahen $35,5 \%$ der Augenärzte eine Zunahme dieses Verhaltens. Aggressionen/Gewalt sind zweifelsohne keine neue Problematik in der Medizin, jedoch wurde dieses Thema erst in den letzten Jahren in den Medien thematisiert. Aus Norwegen liegt eine Studie vor, für welche 1993 sowie 2014 norwegische Ärzte zu Gewalterfahrungen befragt wurden. Dabei zeigte sich interessanterweise keine Zunahme von Gewalterfahrungen [15]. Hier muss allerdings konstatiert werden, dass das skandinavische Gesundheitssystem gänzlich verschieden von dem unsrigen ist.

Die Augenärzte sind in dieser Umfrage der Meinung, dass das nichtärztliche Personal mehr Aggressionen/Gewalt als Ärzte ausgesetzt sei. Dies war auch 

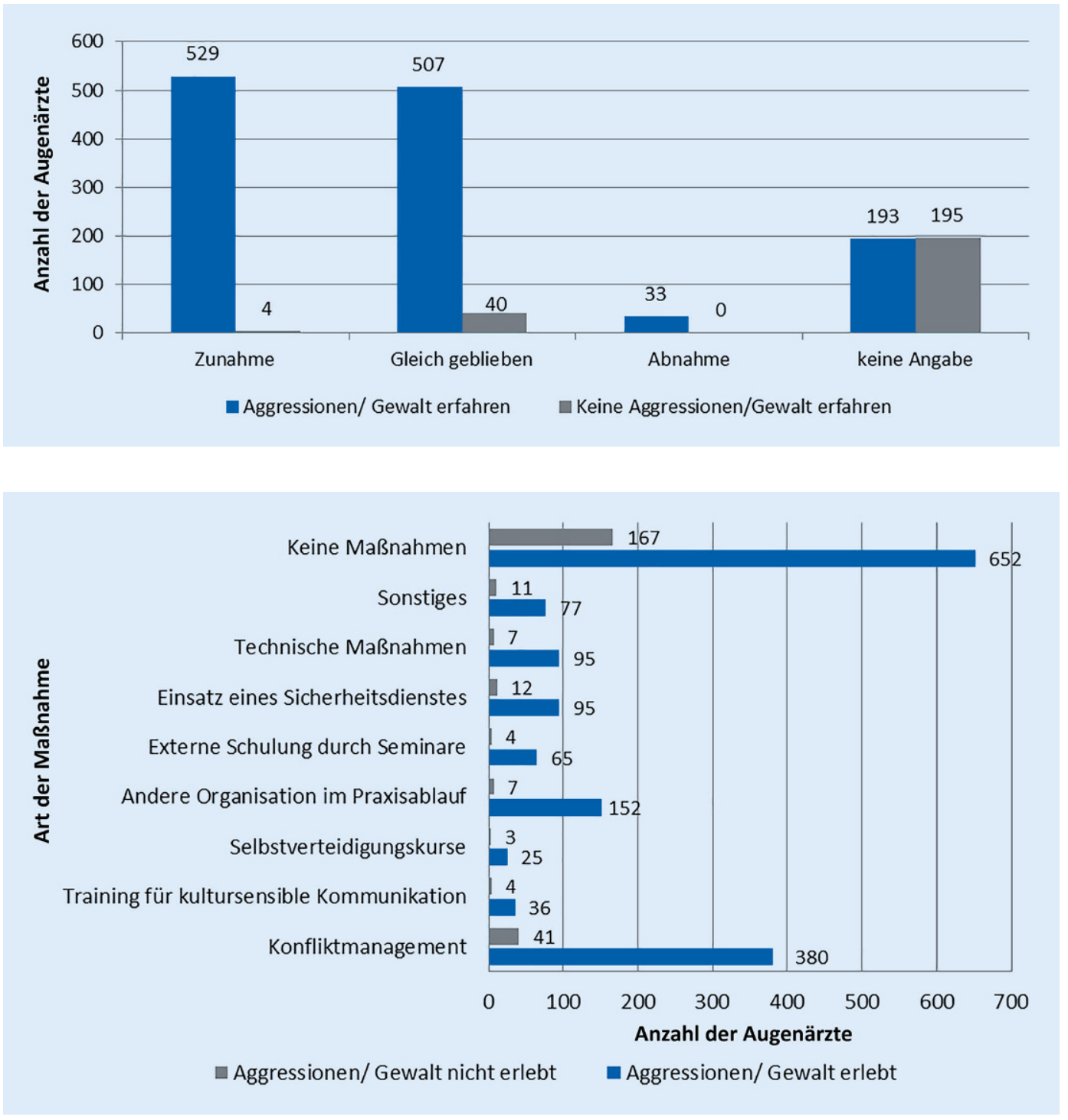

Abb. $1 \triangleleft$ Wie haben sich aggressive/gewalttätige Patientenverhaltensweisen in den letzten 5 Jahren verändert?
Abb. $2<$ Haben Sie in Ihrer Praxis oder in der Klinik Vorkehrungen oder Maßnahmen gegen Gewalt getroffen? Wenn ja, welcher Art? die Einschätzung in der Studie von d'Aubarede et al. [8]. Eine Umfrage unter dem nichtärztlichen Personal in der Augenheilkunde mag daher ebenfalls empfohlen sein.

Als Limitation muss angemerkt werden, dass Ärzte mit Gewalterfahrungen tendenziell eher an der Umfrage teilgenommen haben könnten als diejenigen, die keine Aggressionen erfahren haben, und folglich auch die Zahlen zu hoch ausfallen könnten. Verbale Übergriffe sind zum Teil subjektiv und werden von den Teilnehmern verschieden wahrgenommen. Ärzte, die Deutsch nicht als Muttersprache hatten, gaben seltener Erfahrungen von verbaler Gewalt oder aggressivem Verhalten an, obwohl mit $75,5 \%$ auch bei diesen der Anteil hoch ist. Diese Umfrage zeigte, dass Ärzte mit Migrationshintergrund oder anderer Muttersprache laut eigenen Angaben sogar weniger Gewalt als deutsche Ärzte erlebten $(82,3 \% \mathrm{zu} 84,3 \%)$, obwohl der Migrationshintergrund häufig als Grund für Aggressionen/Gewalt angesehen wurde.

Die Anzahl von Ärzten, die einen vollendeten Suizid während der Behandlung erlebten, ist mit $27(1,8 \%)$ unerwartet hoch. Leider gab es hierzu wenig weitere Informationen in den Freitextfeldern, sodass diese eher nicht nachvollziehbare Aussage unsererseits nicht adäquat bewertet werden kann. Denkbar ist, dass betroffene Augenärzte im Rahmen dieser Umfrage auch über entsprechende Fälle berichteten, die sie zwar in ihrem direkten Umfeld, allerdings nicht im Rahmen der unmittelbaren, selbst durchgeführten Konsultation erlebt hatten. Nur ein Arzt berichtete als schlimmsten Vorfall über einen Patienten, welcher an einer Krebserkrankung litt und sich aus der höchsten Krankenhausetage stürzte.

Da insgesamt mehr Augenärzte in Praxen als in Kliniken arbeiten, liegt es nahe, dass in den Praxen auch mehr Gewalt als in der Klinik erlebt wird. Allerdings zeigte sich kein signifikanter Unterschied zwischen den Gewalterfahrungen von Praxis- und Klinikärzten. Beachtet werden muss dabei, dass auch in der Praxis tätige Ärzte in der Regel früher in Kliniken tätig waren und somit über Vergleichsmöglichkeiten verfügen. 
Im Ärztemonitor 2018 hatten 79\% der Augenärzte keine Präventionsmaßnahmen getroffen [5]. In unserer Auswertung waren es $54 \%$ der Augenärzte, die bislang keine Maßnahmen eingeführt hatten. Dies könnte ein Indiz dafür sein, dass viele Situationen bislang ohne Vorbereitung gehandhabt werden konnten. Einrichtungen versuchen v. a. durch Präventionsmaßnahmen wie Schulungen in Konfliktmanagement und durch Maßnahmen der Praxisorganisation Gewalt vorzubeugen. Dazu gehören etwa eine Verkürzung der Wartezeiten, räumliche Separierung von Patienten oder die Zurverfügungstellung von Beschäftigungsmöglichkeiten für Patienten während der Wartezeit [16]. Aufgrund des hohen Anteils von erlebter Aggression/Gewalt in dieser Umfrage sollten geeignete Maßnahmen zur Gewaltprävention entwickelt und implementiert werden. Eine Möglichkeit wäre es, bereits im Medizinstudium auf die Thematik hinzuweisen und entsprechende Kurse zur Konfliktbewältigung anzubieten. Regelmäßige Kurse, um den Umgang mit „schwierigen“ Patienten zu üben und gestresste Patienten besser zu erkennen, sind sinnvoll. Situationen, in denen Mitarbeiter allein mit den Patienten in Praxen/Einrichtungen sind, sollten vermieden werden oder entsprechende Vorsichtsmaßnahmen getroffen werden wie die Ausrichtung der Behandlungsräume unter Sicherheitsaspekten (z.B. Untersuchungsräume mit 2 Ausgängen bzw. vorgeplante Fluchtwege) oder die Installation von Überwachungskameras oder Notrufknöpfen [17]. Ein bekanntes Konzept zur Konfliktvermeidung ist z. B. das STAMP-Konzept (Akronym für: „Staring and eye contact, Tone and volume of voice, Anxiety, Mumbling and Pacing"), das auf Basis von Beobachtungen in australischen Notaufnahmen entwickelt wurde und Indikatoren, die zu Aggressionen/Gewalt führen können, beschreibt [18]. Die Wiederholung dieser Umfrage im Verlauf z. B. nach 5 Jahren ist sinnvoll, um die Entwicklung darzustellen und weiteren Handlungsbedarf zu erkennen.

\section{Fazit \\ - Insgesamt ist der Anteil von Augen- ärzten in der Umfrage, die Aggressio- nen/Gewalt jemals in ihrer Tätigkeit erfahren haben mit 83,8\% sehr hoch. \\ - Augenärztinnen sowie junge Ärz- tinnen und Ärzte gaben signifikant häufiger Gewalterfahrungen an. \\ - Augenärzte erwarten, dass das medizinische Assistenzpersonal noch mehr Gewalt als Ärzte erfährt. \\ - $54 \%$ der Augenärzte haben bis- lang keine Präventionsmaßnahmen getroffen. \\ - Weitere Diskussionen und Handlun- gen sowohl in der Politik als auch in der Gesellschaft sollten folgen, um die Sicherheit der Mitarbeiter in augenärztlichen Einrichtungen zu verbessern.}

\section{Korrespondenzadresse}

\section{Dr. med. Christina Jacobsen}

Universitätsklinik für Augenheilkunde, Medizinische Hochschule Hannover (MHH) Hannover, Deutschland

jacobsen.christina@mh-hannover.de

Danksagung. Wir bedanken uns bei DOG und BVA für die gute Zusammenarbeit. Zudem bedanken wir uns bei allen Umfrageteilnehmern.

Funding. Open Access funding provided by Projekt DEAL.

\section{Einhaltung ethischer Richtlinien}

Interessenkonflikt. C. Jacobsen, I. Volkmann, F. Wedegärtner, J. Harris, B. Bertram, P. Gass, B. Bambas und C. Framme geben an, dass kein Interessenkonflikt bezüglich des Manuskripts besteht.

Für diesen Beitrag wurden von den Autoren keine Studien an Menschen oder Tieren durchgeführt. Für die aufgeführten Studien gelten die jeweils dort angegebenen ethischen Richtlinien.

Open Access. Dieser Artikel wird unter der Creative Commons Namensnennung 4.0 International Lizenz veröffentlicht, welche die Nutzung, Vervielfältigung, Bearbeitung, Verbreitung und Wiedergabe in jeglichem Medium und Format erlaubt, sofern Sie den/die ursprünglichen Autor(en) und die Quelle ordnungsgemäß nennen, einen Link zur Creative Commons Lizenz beifügen und angeben, ob Änderungen vorgenommen wurden.

Die in diesem Artikel enthaltenen Bilder und sonstiges Drittmaterial unterliegen ebenfalls der genannten Creative Commons Lizenz, sofern sich aus der Abbil- dungslegende nichts anderes ergibt. Sofern das betreffende Material nicht unter der genannten Creative Commons Lizenz steht und die betreffende Handlung nicht nach gesetzlichen Vorschriften erlaubt ist, ist für die oben aufgeführten Weiterverwendungen des Materials die Einwilligung des jeweiligen Rechteinhabers einzuholen.

Weitere Details zur Lizenz entnehmen Sie bitte der Lizenzinformation auf http://creativecommons.org/ licenses/by/4.0/deed.de. 


\section{Anhang}

Tab. 4 Fragebogen

A. Bitte geben Sie an, ob Sie folgende Erfahrungen jemals als Arzt/Ärztin in der Augenheilkunde gemacht haben Beispiele

Nein Ja

Wie oft in den letzten 12 Mona-

1. Verbale Übergriffe ohne Drohung

Beleidigungen, Fluchen, Beschimpfungen, Herumbrüllen, persönliche Beleidigungen, Schreien

ten?

\section{Bedrohendes verbales Verhalten}

3. Demütigendes aggressives Verhalten

4. Herausforderndes aggressives Verhalten

5. Passiv aggressives Verhalten

6. Spaltend aggressives Verhalten

\section{Bedrohlich körperliches Verhalten}

8. Zerstörerisch aggressives Verhalten

9. Mäßige körperliche Gewalt

10. Schwere körperliche Gewalt

11. Mäßige gegen sich selbst gerichtete Gewalt

12. Schwere gegen sich selbst gerichtete Gewalt

13. Versuchter Suizid

14. Vollendeter Suizid

15. Sexuelle Einschüchterung/Belästigung

16. Sexueller Übergriff

17. Wo haben sich die Vorfälle vor allem ereignet?

18. Wann haben sich die Vorfälle vor allem ereignet?

19. Wie haben sich die oben angegebenen Patientenverhaltensweisen in den letzten fünf Jahren verändert?
Boshaft Fluchen, verbale Drohung von Gewalt, Wutanfälle, Androhung von Gewalt außerhalb des Arbeitsplatzes

Eindeutige persönliche Beleidigungen, ausfällig Fluchen, Spucken, abwertende Bemerkungen und Gesten Provokation oder Verhalten, das zu negativer Reaktion führt

Verhalten, das irritierend, störend, blockierend wirkt

Manipulatives Verhalten, das zu Streit oder Disharmonie beim Personal führt

Schlagen von Türen, Werfen und Treten von Gegenständen, Bedrohen mit Waffen

Beschädigen, Zerstören oder Zerschlagen von Gegenständen

Beißen, Schlagen, Treten, Würgen, Austeilen von Fausthieben, Kratzen, an den Haaren Ziehen oder Ausreißen

Schwere Verletzungen, die zu einer ärztlichen Behandlung führten (z. B. Knochenbrüche, Bewusstlosigkeit oder Fleischwunden)

PatientInnen, die sich selbst kratzen, beißen, schlagen etc. mit keinen oder nur kleineren Verletzungen (während der Konsultation)

Behandlungsbedürftige Selbstverstümmelung, tiefe Schnitte, Bisse, Verbrennungen, innere Verletzungen, Brüche, Bewusstlosigkeit (während der Konsultation)

Einnahme einer Medikamentenüberdosis, Aufschneiden der Pulsadern, Sprung von Gebäuden (während der Konsultation)

(Während der Konsultation)

Obszöne Gesten, exhibitionistische Übergriffe, zweideutige Bemerkungen, private Kontaktaufnahme, sexistische Verhaltensweisen

Sexuelle Nötigung, Vergewaltigung

$\square$ Praxis

$\square$ Klinik

$\square$ Heimbesuche

$\square$ Hausbesuche

$\square$ Entfällt

$\square$ Reguläre Arbeitszeit

$\square$ Nachts

$\square$ Wochenende/Feiertag

$\square$ Notdienst

$\square$ Entfällt

$\square$ Sie haben zugenommen

$\square$ Sie sind gleich geblieben

$\square$ Sie haben abgenommen

$\square$ Keine Angabe

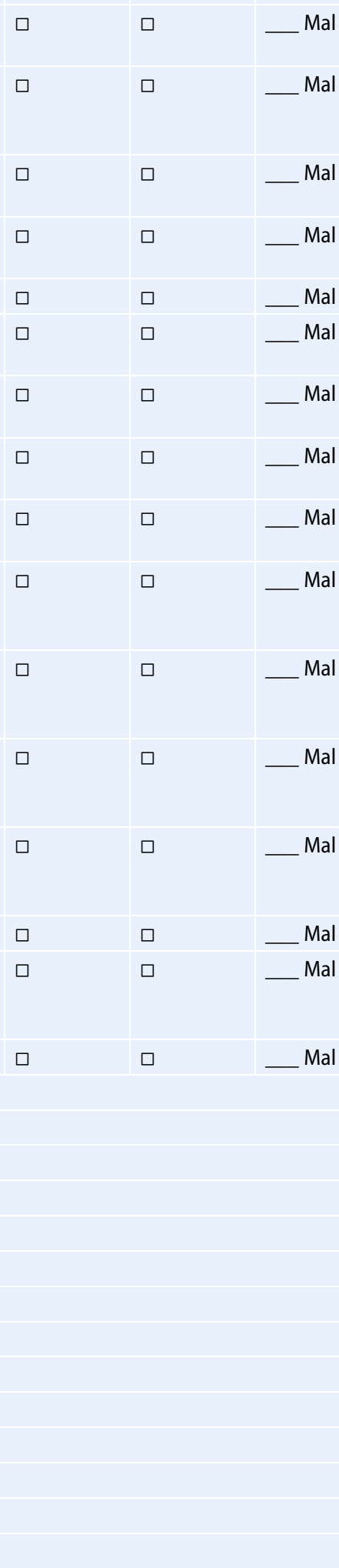




\section{Originalien}

Tab. 4 (Fortsetzung)

B. Teilnahme am Notdienst

20. Nehmen Sie am Notdienst am

Wochenende oder außerhalb regulärer

Dienstzeiten teil?

$\square$ Nein $\rightarrow$ Weiter zu Teil C

$\square \mathrm{Ja}$

21. Sind Sie im Notdienst allein?

$\square \mathrm{Ja}$

$\square$ Nein, ein ärztlicher Kollege unterstützt mich

$\square$ Nein, eine medizinische Fachkraft unterstützt mich

$\square$ Sonstiges:

20. Wie lange warten die Patienten

$\square$ Unter $30 \mathrm{~min}$

außerhalb der regulären Sprechzeiten

$\square$ Zwischen 30 und $60 \mathrm{~min}$

$\square$ Zwischen 60 und 120 min

$\square$ Zwischen 120 und $180 \mathrm{~min}$

$\square$ Länger als $180 \mathrm{~min}$

C. Gewalt gegenüber Assistenzpersonal (wie Pflegekraft, MFA)
23. Haben Sie oben genannte
Gewalterfahrungen bei Ihrem
Assistenzpersonal miterlebt bzw. wurde
Ihnen davon berichtet?
$\square \mathrm{Ja}$
$\square$ Nein
24. Was war aus den oben genannten Kate-
$\square$ Keine Angabe
(Drop down) gorien die schlimmste Gewalterfahrung, die dem Assistenzpersonal widerfuhr?

25. Haben Sie den Eindruck, dass nichtärztliches Personal mehr Gewalt als Ärzte/Ärztinnen erfährt?

\section{Angaben zu Ihrer Person und Praxis}

26. Wie alt sind Sie?

27. Welches Geschlecht haben Sie?

$\square$ Ja, das Assistenzpersonal erfährt mehr Gewalt

$\square$ Nein, das ärztliche Personal erlebt mehr Gewalt

$\square$ Es existiert kein Unterschied

$\square$ Keine Angabe

$\square$ Männlich
$\square$ Weiblich

28. In welchem Bundesland arbeiten Sie?

29. Arbeiten Sie in einer Stadt mit mehr als 50.000 Einwohnern?

$\square \mathrm{Ja}$

$\square$ Nein

$\square$ Keine Angabe

30. Haben Sie einen Migrationshintergrund?

$\square \mathrm{Ja}$

$\square$ Nein

$\square$ Keine Angabe

31. Ist Deutsch Ihre Muttersprache?

$\square$ Ja

32. Haben Sie den Eindruck, dass Sie aufgrund Ihrer Sprache/Herkunft Ziel von Patientenaggressionen wurden?

33. In welcher Einrichtung arbeiten Sie aktuell?

$\square$ Nein, sondern:

$\square \mathrm{Ja}$

$\square$ Nein

$\square$ Keine Angabe

$\square$ Einzelpraxis

$\square$ Gemeinschaftspraxis

$\square$ Medizinisches Versorgungszentrum

$\square$ Klinik

$\square$ Universitätsklinik 
Tab. 4 (Fortsetzung)

34. Welche Funktion haben Sie aktuell?

35. Haben Sie in Ihrer Praxis oder in der Klinik Vorkehrungen oder Maßnahmen gegen Gewalt getroffen? Wenn ja, welcher Art? $\square$ Assistenzarzt/-ärztin im 1. oder 2. Weiterbildungsjahr

$\square$ Assistenzarzt/-ärztin ab dem 3. Weiterbildungsjahr

$\square$ Facharzt/-ärztin

$\square$ Oberarzt/-ärztin

$\square$ Leitender Oberarzt/-ärztin

$\square$ Chefarzt/-ärztin

$\square$ Konfliktmanagement für Ärzte/Mitarbeiter (interne Schulung, Leitlinien)

$\square$ Training für kultursensible Kommunikation

$\square$ Selbstverteidigungskurse

$\square$ Andere Organisation im Praxisablauf

$\square$ Externe Schulung durch Seminare (Anti-Gewalt- oder Deeskalationstraining)

$\square$ Einsatz eines Sicherheitsdienstes

$\square$ Technische Maßnahmen (Videoüberwachung, Panikknopf)

$\square$ Nein

\section{Literatur}

1. Schindler J (2019) Die enthemmte Gesellschaft. Spiegel 2019(12):10-14

2. DeutscherÄrzteverlag GmbH,ÄrzteblattRD (2019) Bundesärztekammer pocht auf mehr Schutz für Ärzte und Helfer. https://www.aerzteblatt. de/nachrichten/97387/Bundesaerztekammerpocht-auf-mehr-Schutz-fuer-Aerzte-und-Helfer. Zugegriffen: 14. Juli 2019

3. Vorderwülbecke F, Feistle M, Mehring M, Schneider A, Linde K (2015) Aggression and violence against primary care physicians-a nationwide questionnaire survey. Dtsch Arztebl Int 112(10):159-165. https://doi.org/10.3238/ arztebl.2015.0159

4. Ruhruniversität Bochum (2018) Gewalt gegen Einsatzkräfte der Feuerwehren und Rettungsdienste in Nordrhein-Westfalen. https://www.sicherer-rettungsdienst.de/media/ docs/Abschlussbericht_Gewalt\%20gegen \%20Einsatzkr\%C3\%A4fte.pdf.Zugegriffen: 14.Juli 2019

5. Kassenärztliche Bundesvereinigung (KBV) (2018) Tabellenband Ärztemonitor 2018 Ergebnisse für Haus- und Fachärzte. https://www.kbv.de/ html/aerztemonitor.php (Erstellt: 16. Okt. 2018). Zugegriffen: 14. Juli 2019

6. ALBashtawy M, Aljezawi M (2016) Emergency nurses' perspective of workplace violence in Jordanian hospitals: a national survey. Int Emerg Nurs 2016(24):61-65. https://doi.org/10.1016/j. ienj.2015.06.005

7. Cannavò M, La Torre F, Sestili C, La Torre G, Fioravanti M (2019) Work related violence as a predictor of stress and correlated disorders in emergency department healthcare professionals. Clin Ter 170(2):e110-e123. https://doi.org/10. 7417/CT.2019.2120

8. d'Aubarede C, Sarnin P, Cornut P-L et al (2016) Impacts of users' antisocial behaviors in an ophthalmologic emergency department-a qualitative study. J Occup Health 58(1):96-106. https://doi.org/10.1539/joh.15-0184-FS

9. Oud N (2000) POPAS (Perception of Prevalence of Aggression Scale) CONNECTING, Partnerschaftsunternehmen für Beratung und Ausbildung, Hakfort 621, 1102 LA Amsterdam - Niederlande. http://www.gesundheitsdienstportal.de/risiko- uebergriff/infoplus/6_4_3b.pdf. Zugegriffen: 23. Januar 2020

10. Harwood RH (2017) How to deal with violent and aggressive patients in acute medical settings. J $\mathrm{R}$ Coll Physicians Edinb 47(2):94-101. https://doi. org/10.4997/JRCPE.2017.218

11. Schuster AK, Wolfram C, Pfeiffer N, Finger RP (2019) Augenheilkunde 2019 - Wo stehen wir?: Eine Betrachtung der Versorgungssituation in Deutschland. Ophthalmologe 116(9):829-837. https://doi.org/10.1007/s00347-019-0894-2

12. Richter-Kuhlmann E (2019) Arbeitsbedingungen im Krankenhaus: Burn-outschon beim Nachwuchs. Dtsch Arztebl Int 116(48):A-2222

13. Kumar M, Verma M, Das T, Pardeshi G, Kishore J, Padmanandan A (2016) A study of Workplace violence experienced by doctors and associated risk factors in a tertiary care hospital of South Delhi, India. J Clin Diagn Res 10(11):LC6-LC10. https:// doi.org/10.7860/JCDR/2016/22306.8895

14. Cabrera MT, Enyedi LB, Ding L, MacDonald SM (2019) Sexual harassment in ophthalmology: a survey study. Ophthalmology 126(1):172-174. https://doi.org/10.1016/j.ophtha.2018.09.016

15. Johansen IH, Baste V, Rosta J, Aasland OG, Morken T (2017) Changes in prevalence of workplace violenceagainst doctors in allmedical specialties in Norway between 1993 and 2014: a repeated crosssectional survey. Bmj Open 7(8):e17757-2017. https://doi.org/10.1136/bmjopen-2017-017757

16. Sonnenmoser M (2017) Der schwierige Patient: Aggressivität und Gewalt - Der Respekt nimmt ab. Dtsch Arztebl Int 114(10):A-482

17. Berlanda S, Pedrazza M, Fraizzoli M, de Cordova F (2019) Addressing risks of violence against healthcare staff in emergency departments: the effects of job satisfaction and attachment style. Biomed Res Int 2019(2019):5430870. https://doi. org/10.1155/2019/5430870

18. Luck L, Jackson D, Usher K (2007) STAMP: Components of observable behaviourthatindicate potential for patient violence in emergency departments. J Adv Nurs 59(1):11-19. https://doi. org/10.1111/j.1365-2648.2007.04308.x 